\title{
On some inequalities relative to the Pompeiu-Chebyshev functional
}

Daniel lanoşi $1^{1 *}$ and Adonia-Augustina Opriş ${ }^{1}$

\section{"Correspondence:}

dan.ianosi@yahoo.com

'Department of Mathematics,

Technical University of Cluj-Napoca,

Cluj-Napoca, Romania

\begin{abstract}
In this paper we study the utility of the functional Pompeiu-Chebyshev in some inequalities. Some results obtained by Alomari will be generalized regarding inequalities with Pompeiu-Chebyshev type functionals, in which linear and positive functionals intervene. We investigate some new inequalities of Grüss type using Pompeiu's mean value theorem. Improvement of known inequalities is also given.
\end{abstract}

Keywords: Chebyshev functional; Pompeiu's mean value theorem; CBS inequality; Grüss inequality

\section{Introduction}

If $f, g$ are integrable functions on $[a, b]$, then the functional defined by

$$
\mathcal{T}(f, g)=\frac{1}{b-a} \int_{a}^{b} f(t) g(t) d t-\frac{1}{b-a} \int_{a}^{b} f(t) d t \cdot \frac{1}{b-a} \int_{a}^{b} g(t) d t
$$

is known as functional Chebyshev, with multiple applications in numerical analysis and probability theory (see [3]).

The following theorem combines a series of results regarding the bounds for this functional.

Theorem 1.1 (See $[3,5-7]$ ) Let $f, g:[a, b] \rightarrow \mathbb{R}$ be two absolutely continuous functions, then

$$
|\mathcal{T}(f, g)| \leq\left\{\begin{array}{l}
\frac{(b-a)^{2}}{12}\left\|f^{\prime}\right\|_{\infty}\left\|g^{\prime}\right\|_{\infty}, \quad \text { iff } f^{\prime}, g^{\prime} \in L_{\infty}[a, b], \\
\frac{1}{4}\left(M_{f}-m_{f}\right)\left(M_{g}-m_{g}\right), \quad \text { if } m_{f} \leq f \leq M_{f} \text { and } m_{g} \leq g \leq M_{g} \\
\frac{b-a}{\pi^{2}}\left\|f^{\prime}\right\|_{2}\left\|g^{\prime}\right\|_{2}, \quad \text { iff }, g^{\prime} \in L_{2}[a, b], \\
\frac{1}{8}(b-a)\left(M_{f}-m_{f}\right)\left\|g^{\prime}\right\|_{\infty}, \quad \text { if } m_{f} \leq f \leq M_{f} \text { and } g^{\prime} \in L_{\infty}[a, b]
\end{array}\right.
$$

In [9], Pompeiu established the following mean value theorem for functions defined on an interval $[a, b]$ such that $0 \notin[a, b]$.

(c) The Author(s) 2020. This article is licensed under a Creative Commons Attribution 4.0 International License, which permits use sharing, adaptation, distribution and reproduction in any medium or format, as long as you give appropriate credit to the original author(s) and the source, provide a link to the Creative Commons licence, and indicate if changes were made. The images or other third party material in this article are included in the article's Creative Commons licence, unless indicated otherwise in a credit line to the material. If material is not included in the article's Creative Commons licence and your intended use is not permitted by statutory regulation or exceeds the permitted use, you will need to obtain permission directly from the copyright holder. To view a copy of this licence, visit http://creativecommons.org/licenses/by/4.0/. 
Theorem 1.2 For every function $f \in C^{1}[a, b], 0 \notin[a, b]$ and for all $x, y \in[a, b], x \neq y$, there is $c \in(x, y)$ such that

$$
\frac{x f(y)-y f(x)}{x-y}=f(c)-c f^{\prime}(c)
$$

From (3), we obtain that

$$
|x f(y)-y f(x)| \leq|x-y|\left\|f-e_{1} f^{\prime}\right\|,
$$

where $e_{i}=x^{i}, i=\overline{0, n}, n \in \mathbb{N}$.

In 2005, Pachpatte (see [8]) introduced the following functional.

If $f, g:[a, b] \rightarrow \mathbb{R}$ are two differentiable functions on $(a, b)$, then

$$
\mathcal{P}(f, g)=\int_{a}^{b} f(x) g(x) d x-\frac{3}{b^{3}-a^{3}} \int_{a}^{b} x f(x) d x \int_{a}^{b} x g(x) d x
$$

and proved the following result.

Theorem 1.3 Iff, $g:[a, b] \rightarrow \mathbb{R}$ are two continuous on $[a, b]$ and differentiable functions on $(a, b)$ such that $0 \notin[a, b]$, then

$$
|\mathcal{P}(f, g)| \leq(b-a)\left(1-\frac{3}{4} \frac{(a+b)^{2}}{a^{2}+a b+b^{2}}\right)\left\|f-e_{1} f^{\prime}\right\|_{\infty}\left\|g-e_{1} g^{\prime}\right\|_{\infty}
$$

Dragomir (see [4]) studied the Pompeiu-Chebyshev functional and changed it as follows:

$$
\widehat{\mathcal{P}}(f, g)=\frac{b^{3}-a^{3}}{3} \mathcal{P}(f, g) .
$$

The following result, obtained by Dragomir in [4], will be used in some demonstrations included in this paper.

Lemma 1.4 Let $f:[a, b] \rightarrow \mathbb{R}$ be an absolutely continuous function on $[a, b], b>a>0$. Then, for any $x, y \in[a, b]$, we have

$$
|y f(x)-x f(y)| \leq\left\{\begin{array}{l}
|x-y||| f-e_{1} f^{\prime} \|_{\infty}, \quad \text { iff }-e_{1} f^{\prime} \in L^{\infty}[a, b], \\
\left(\frac{1}{2 q-1}\right)^{\frac{1}{q}}\left|\frac{x^{q}}{y^{q-1}}-\frac{y^{q}}{x^{q-1}}\right|^{\frac{1}{q}}|| f-e_{1} f^{\prime} \|_{p}, \\
i f f-e_{1} f^{\prime} \in L^{p}[a, b], \\
\left\|f-e_{1} f^{\prime}\right\|_{1} \frac{\max \{x, y\}}{\min \{x, y\}}, \quad \text { iff }-e_{1} f^{\prime} \in L^{1}[a, b],
\end{array}\right.
$$

where $p, q>1$ with $\frac{1}{p}+\frac{1}{q}=1$.

In [1], Alomari studied and generalized some inequalities related to the PompeiuChebyshev functional.

The purpose of this paper is to generalize the results of Alomari considering the Pompeiu-Chebyshev functional in which linear and positive functionals intervene. 


\section{Main results}

In the following we denote by $\mathcal{F}$ a set of linear functions defined on the interval $I=[a, b]$. We will assume that the set $\mathcal{F}$ contains the constant and polynomial functions, and we suppose that, if $f, g \in \mathcal{F}$, then $f \cdot g \in \mathcal{F}$.

Definition 2.1 Let $A, B: \mathcal{F} \rightarrow \mathbb{R}$ be two linear and positive functionals.

If $f, g \in \mathcal{F}$, we denote

$$
\mathcal{P}_{A, B}(f, g)=\frac{1}{2}\left[B\left(e_{2}\right) A(f g)+A\left(e_{2}\right) B(f g)-A\left(e_{1} f\right) B\left(e_{1} g\right)-A\left(e_{1} g\right) B\left(e_{1} f\right)\right] .
$$

We call the functional $\mathcal{P}_{A, B}(f, g)$ a Pompeiu-Chebyshev functional.

Remark 2.2 For any two linear and positive functionals $A, B: \mathcal{F} \rightarrow \mathbb{R}$, we have

$$
\mathcal{P}_{A, B}(f, g)=\mathcal{P}_{B, A}(f, g)
$$

Remark 2.3 If we take

$$
A(f)=B(f)=\int_{a}^{b} f(x) d x, \quad \mathcal{F}=L[a, b],
$$

then the functional $\mathcal{P}_{A, B}(f, g)$ becomes the functional that was studied by Dragomir in [4].

Theorem 2.4 If $\mathcal{F}=C^{1}[a, b], 0 \notin[a, b]$, then

$$
\begin{aligned}
\left|\mathcal{P}_{A, B}(f, g)\right| \leq & \frac{1}{2}\left[B\left(e_{0}\right) A\left(e_{2}\right)+A\left(e_{0}\right) B\left(e_{2}\right)-2 A\left(e_{1}\right) B\left(e_{1}\right)\right] \\
& \times\left\|f-e_{1} f^{\prime}\right\|_{\infty}\left\|g-e_{1} g^{\prime}\right\|_{\infty} .
\end{aligned}
$$

Proof From Lemma 1.4 we have

$$
|x f(y)-y f(x)||x g(y)-y g(x)| \leq(x-y)^{2}\left\|f-e_{1} f^{\prime}\right\|_{\infty}\left\|g-e_{1} g^{\prime}\right\|_{\infty} .
$$

Next, by $A_{x}$ or $B_{y}$ we will understand that the functional $A$, respectively $B$, acts on the variable $x$, respectively $y$.

It is easy to see that

$$
\mathcal{P}_{A, B}(f, g)=\frac{1}{2} A_{x} B_{y}((y f(x)-x f(y))(y g(x)-x g(y))) .
$$

From relations (9) and (10) we get the following:

$$
\left|\mathcal{P}_{A, B}(f, g)\right| \leq \frac{1}{2} A_{x} B_{y}(x-y)^{2} \cdot\left\|f-e_{1} f^{\prime}\right\|_{\infty}\left\|g-e_{1} g^{\prime}\right\|_{\infty} .
$$

Further we have

$$
A_{x} B_{y}\left((x-y)^{2}\right)=B\left(e_{0}\right) A\left(e_{2}\right)+A\left(e_{0}\right) B\left(e_{2}\right)-2 A\left(e_{1}\right) B\left(e_{1}\right) .
$$

Combining relations (11) and (12), we get (8). 
Corollary 2.5 If we take $A(f)=B(f)=\int_{a}^{b} f(x) d x$, then we obtain

$$
\left|\mathcal{P}_{A, B}(f, g)\right| \leq \frac{1}{2}\left[2 \int_{a}^{b} x^{2} d x \int_{a}^{b} d x-2\left(\int_{a}^{b} x d x\right)^{2} d x\right]\left\|f-e_{1} f^{\prime}\right\|_{\infty}\left\|g-e_{1} g^{\prime}\right\|_{\infty}
$$

so,

$$
\left|\mathcal{P}_{A, B}(f, g)\right| \leq \frac{1}{12}(b-a)^{4}\left\|f-e_{1} f^{\prime}\right\|_{\infty}\left\|g-e_{1} g^{\prime}\right\|_{\infty},
$$

which is the inequality obtained by Dragomir in [4].

Definition 2.6 Let $f, g \in \mathcal{F}$. The functions $f$ and $g$ are called synchronous (or similarly ordered) if for all $x, y \in I$, where $I$ is the domain for $f$ and $g$, we have

$$
(f(x)-f(y))(g(x)-g(y)) \geq 0,
$$

and $f$ and $g$ are called asynchronous (or oppositely ordered) if for all $x, y \in I$ we have

$$
(f(x)-f(y))(g(x)-g(y)) \leq 0 .
$$

Theorem 2.7 Let $f, g \in \mathcal{F}$, where $f, g: I \rightarrow \mathbb{R}$ such that $0 \notin I$.

(i) If $\frac{f}{e_{1}}$ and $\frac{g}{e_{1}}$ are synchronous functions, then $\mathcal{P}_{A, B}(f, g) \geq 0$.

(ii) If $\frac{f}{e_{1}}$ and $\frac{g}{e_{1}}$ are asynchronous functions, then $\mathcal{P}_{A, B}(f, g) \leq 0$.

Proof Since $\frac{f}{e_{1}}$ and $\frac{g}{e_{1}}$ are synchronous (asynchronous) functions, we have

$$
\begin{aligned}
& \left(\frac{f(x)}{x}-\frac{f(y)}{y}\right)\left(\frac{g(x)}{x}-\frac{g(y)}{y}\right) \geq(\leq) 0 \\
& \Leftrightarrow \quad(y f(x)-x f(y))(y g(x)-x g(y)) \geq(\leq) 0, \quad \forall x, y \in I .
\end{aligned}
$$

So,

$$
\mathcal{P}_{A, B}(f, g)=\frac{1}{2} A_{x}\left(B_{y}(f, g)\right)=\frac{1}{2} A_{x} B_{y}(y f(x)-x f(y))(x g(y)-y g(x)) \geq(\leq) 0 .
$$

Remark 2.8 If $A(f)=B(f)=\int_{a}^{b} f(x) d x$, then we get Theorem 6 and Corollary 1 from [1].

The following theorem shows a pre-Grüss inequality for the functional $\mathcal{P}_{A, B}(f, g)$ (see [5]).

Theorem 2.9 Let $f, g \in \mathcal{F}$, where $f, g: I \rightarrow \mathbb{R}$. Then

$$
\left|\mathcal{P}_{A, B}(f, g)\right| \leq 1 \cdot\left|\mathcal{P}_{A, B}(f, f)\right|^{\frac{1}{2}}\left|\mathcal{P}_{A, B}(g, g)\right|^{\frac{1}{2}} .
$$

Constant 1 is the best possible.

Proof From the equality

$$
\mathcal{P}_{A, B}(f, g)=\frac{1}{2} A_{x}\left(B_{y}(f, g)\right)=\frac{1}{2} A_{x} B_{y}((x f(y)-y f(x))(x g(y)-y g(x)))
$$


and from the CBS-inequality, we obtain

$$
\left|\mathcal{P}_{A, B}(f, g)\right| \leq\left[\frac{1}{2} A_{x} B_{y}(x f(y)-y f(x))^{2}\right]^{\frac{1}{2}}\left[\frac{1}{2} A_{x} B_{y}(x g(y)-y g(x))^{2}\right]^{\frac{1}{2}} .
$$

But we have

$$
\frac{1}{2} A_{x} B_{y}(x f(y)-y f(x))^{2}=\mathcal{P}_{A, B}(f, f)
$$

and

$$
\frac{1}{2} A_{x} B_{y}(x g(y)-y g(x))^{2}=\mathcal{P}_{A, B}(g, g) .
$$

From the above the conclusion is obtained.

We notice that for $f(x)=g(x)=c \cdot x-1, c \in \mathbb{R}$, fixed we obtain the equality in (16).

We note that for $A(f)=B(f)=\int_{a}^{b} f(x) d x$ we get Theorem 7 from [1].

Theorem 2.10 Let $f, g:[a, b] \rightarrow \mathbb{R}, 0<a<b, f, g \in \mathcal{F}$. If there exist real numbers $m_{f}$, $M_{f}, m_{g}, M_{g}$ such that $m_{f} \leq f(x) \leq M_{f}$ and $m_{g} \leq g(x) \leq M_{g}, \forall x \in[a, b]$, then the following inequality

$$
\begin{aligned}
\left|\mathcal{P}_{A, B}(f, g)\right| \leq & \frac{1}{2}\left(A\left(e_{2}\right) B\left(e_{0}\right)+B\left(e_{2}\right) A\left(e_{0}\right)-2 A\left(e_{1}\right) B\left(e_{1}\right)\right) \\
& \times\left(b M_{f}-a m_{f}\right)\left(b M_{g}-a m_{g}\right)
\end{aligned}
$$

holds.

Proof From the assumptions of the theorem we have

$$
a m_{f} \leq y f(x) \leq b M_{f} \quad \text { and } \quad-b M_{f} \leq-x f(y) \leq-a m_{f} .
$$

Adding the last inequalities, we have

$$
a m_{f}-b M_{f} \leq y f(x)-x f(y) \leq b M_{f}-a m_{f}
$$

or

$$
|y f(x)-x f(y)| \leq b M_{f}-a m_{f}
$$

In the same way we proceed for the function $g$, and we get

$$
\begin{aligned}
& |x g(y)-y g(x)| \leq b M_{g}-a m_{g}, \\
& |x f(y)-y f(x)| \leq b M_{f}-a m_{f} .
\end{aligned}
$$

From (18) we get

$$
|(x f(y)-y f(x))(x g(y)-y g(x))| \leq\left(b M_{f}-a m_{f}\right)\left(b M_{g}-a m_{g}\right) .
$$


So, we have

$$
\left|\mathcal{P}_{A, B}(f, g)\right| \leq\left(b M_{f}-a m_{f}\right)\left(b M_{g}-a m_{g}\right) \mathcal{P}_{A, B}(1,1)
$$

Since

$$
\mathcal{P}_{A, B}(1,1)=\frac{1}{2}\left(A\left(e_{2}\right) B\left(e_{0}\right)+B\left(e_{2}\right) A\left(e_{0}\right)-2 A\left(e_{1}\right) B\left(e_{1}\right)\right),
$$

we get the inequality from the conclusion.

Corollary 2.11 If we take $A(f)=B(f)=\int_{a}^{b} f(x) d x$, then we get the following inequality:

$$
\left|\mathcal{P}_{A, B}(f, g)\right| \leq \frac{1}{12}(b-a)^{4}\left(b M_{f}-a m_{f}\right)\left(b M_{g}-a m_{g}\right)
$$

Theorem 2.12 Let $A, B: \mathcal{F} \rightarrow \mathbb{R}$ be two linear and positive functionals. Let $f, g:[a, b] \rightarrow$ $\mathbb{R}, 0<a<b, f, g \in \mathcal{F}$. If there exist real numbers $m_{f}, M_{f}, m_{g}, M_{g}$ such that $m_{f} \leq f(x) \leq M_{f}$ and $m_{g} \leq g(x) \leq M_{g}, \forall x \in[a, b]$, then the following inequality holds:

$$
\left|\mathcal{P}_{A, B}(f, g)\right| \leq \frac{1}{2}\left(b M_{f}-a m_{f}\right)\left(b M_{g}-a m_{g}\right) A_{x} B_{y}(1)
$$

Proof From the assumptions of the theorem we have

$$
a m_{f} \leq y f(x) \leq b M_{f} \quad \text { and } \quad-b M_{f} \leq-x f(y) \leq-a m_{f} .
$$

Adding the last inequalities, we have

$$
a m_{f}-b M_{f} \leq y f(x)-x f(y) \leq b M_{f}-a m_{f}
$$

or

$$
|y f(x)-x f(y)| \leq b M_{f}-a m_{f}
$$

In the same way we proceed for the function $g$, and we get

$$
|x g(y)-y g(x)| \leq b M_{g}-a m_{g}
$$

From the above we get

$$
|(x f(y)-y f(x))(x g(y)-y g(x))| \leq\left(b M_{f}-a m_{f}\right)\left(b M_{g}-a m_{g}\right)
$$

and then

$$
\left|\mathcal{P}_{A, B}(f, g)\right| \leq \frac{1}{2} A_{x} B_{y}\left(\left(b M_{f}-a m_{f}\right)\left(b M_{g}-a m_{g}\right)\right) .
$$

The last inequality is equivalent to the conclusion. 
Remark 2.13 If we take $A(f)=B(f)=\int_{a}^{b} f(x) d x$, then we get Theorem 8 from [1].

Theorem 2.14 Let $f, g:[a, b] \rightarrow \mathbb{R}, 0<a<b, f, g \in \mathcal{F}$. If there exist real numbers $m_{g}, M_{g}$ such that $m_{g} \leq g(x) \leq M_{g}, \forall x \in[a, b]$, then the following inequality

$$
\begin{aligned}
\left|\mathcal{P}_{A, B}(f, g)\right| \leq & \frac{1}{\sqrt{2}}\left[A\left(e_{2}\right) B\left(e_{0}\right)+A\left(e_{0}\right) B\left(e_{2}\right)-2 A\left(e_{1}\right) B\left(e_{1}\right)\right]^{\frac{1}{2}}\left|\mathcal{P}_{A, B}(f, f)\right|^{\frac{1}{2}} \\
& \times\left(b M_{g}-a m_{g}\right)
\end{aligned}
$$

holds.

Proof From (16) we get

$$
\left|\mathcal{P}_{A, B}(f, g)\right| \leq \sqrt{\mathcal{P}_{A, B}(f, f) \cdot \mathcal{P}_{A, B}(g, g)}
$$

From (10) and (17) we have

$$
\left|\mathcal{P}_{A, B}(g, g)\right| \leq \frac{1}{2}\left(A\left(e_{2}\right) B\left(e_{0}\right)+A\left(e_{2}\right) B\left(e_{0}\right)-2 A\left(e_{1}\right) B\left(e_{1}\right)\right)\left(b M_{g}-a m_{g}\right)^{2}
$$

From relationships (22) and (23) we get the conclusion.

Corollary 2.15 If we take $A(f)=B(f)=\int_{a}^{b} f(x) d x$, then we obtain the following inequality:

$$
\left|\mathcal{P}_{A, B}(g, g)\right| \leq \frac{1}{2 \sqrt{3}}(b-a)^{2}\left(b M_{g}-a m_{g}\right)\left|\mathcal{P}_{A, B}(f, f)\right|^{\frac{1}{2}}
$$

Theorem 2.16 Let $A, B: \mathcal{F} \rightarrow \mathbb{R}$ be two linear and positive functionals. Let $f, g:[a, b] \rightarrow$ $\mathbb{R}, 0<a<b, f, g \in \mathcal{F}$. If there exist real numbers $m_{g}, M_{g}$ such that $m_{g} \leq g(x) \leq M_{g}, \forall x \in$ $[a, b]$, then the following inequality holds:

$$
\left|\mathcal{P}_{A, B}(f, g)\right| \leq \frac{1}{\sqrt{2}}\left|\mathcal{P}_{A, B}(f, f)\right|^{\frac{1}{2}}\left|A_{x} B_{y}(1)\right|^{\frac{1}{2}}\left(b M_{g}-a m_{g}\right) .
$$

Proof From (16) we get

$$
\left|\mathcal{P}_{A, B}(f, g)\right| \leq \sqrt{\mathcal{P}_{A, B}(f, f) \cdot \mathcal{P}_{A, B}(g, g)}
$$

From (17) for $f=g$ we have

$$
\left|\mathcal{P}_{A, B}(g, g)\right| \leq \frac{1}{2}\left(b M_{g}-a m_{g}\right)^{2} A_{x} B_{y}(1)
$$

Replacing (27) in (26), we get the conclusion.

Remark 2.17 If we take $A(f)=B(f)=\int_{a}^{b} f(x) d x$, then we get Theorem 9 from [1].

An improvement of inequality (17) from Theorem 2.10 is given below. 
Theorem 2.18 Let $D$ be a subset of the real line such that $D \subset[a, b], a>0$. If $f, g \in \mathcal{F}$, $f, g: D \rightarrow \mathbb{R}, 0<a<b$ and we suppose that there exist real numbers $m_{f}, M_{f}, m_{g}, M_{g}$ such that $m_{f} \leq f(x) \leq M_{f}$ and $m_{g} \leq g(x) \leq M_{g}, \forall x \in D$, then the following inequality holds:

$$
\left|\mathcal{P}_{A, B}(f, g)\right| \leq \frac{1}{2} A_{x} B_{y}\left(\left|x M_{f}-y m_{f}\right|\left|x M_{g}-y m_{g}\right|\right) .
$$

Proof It is easy to see, in these conditions, that we have

$$
\begin{aligned}
& |x f(y)-y f(x)| \leq\left|x M_{f}-y m_{f}\right|, \\
& |x g(y)-y g(x)| \leq\left|x M_{g}-y m_{g}\right| .
\end{aligned}
$$

From the above we obtain

$$
|(x f(y)-y f(x))(x g(y)-y g(x))| \leq\left|\left(x M_{f}-y m_{f}\right)\left(x M_{g}-y m_{g}\right)\right|, \quad \forall x, y \in D .
$$

Applying the linear and positive functional $A_{x} B_{y}$ and considering that

$$
\left|A_{x} B_{y}(h(x, y))\right| \leq A_{x} B_{y}(|h(x, y)|),
$$

the statement results.

Remark 2.19 If we take $A(f)=B(f)=\int_{a}^{b} f(x) d x$, then from (22) we get Theorem 10 from [1].

A generalization of this is given in what follows.

Theorem 2.20 Let $f, g: D \rightarrow \mathbb{R}, D \subset[a, b], 0<a<b, f, g \in \mathcal{F}$. If there exist real numbers $m_{f}, M_{f}, m_{g}, M_{g}$ such that $m_{f} \leq f(x) \leq M_{f}$ and $m_{g} \leq g(x) \leq M_{g}, \forall x \in D$, then the following inequality holds:

$$
\left|\mathcal{P}_{A, B}(f, g)\right| \leq \frac{1}{4} K_{f} K_{g},
$$

where

$$
\begin{aligned}
K_{f}= & {\left[\left(M_{f}^{2}+m_{f}^{2}\right)\left(A\left(e_{0}\right) B\left(e_{2}\right)+B\left(e_{0}\right) A\left(e_{2}\right)\right)\right.} \\
& \left.+\left(M_{f}^{2}-m_{f}^{2}\right)\left|A\left(e_{0}\right) B\left(e_{2}\right)-B\left(e_{0}\right) A\left(e_{2}\right)\right|-4 A\left(e_{1}\right) B\left(e_{1}\right) m_{f} M_{f}\right]^{\frac{1}{2}}
\end{aligned}
$$

and

$$
\begin{aligned}
K_{g}= & {\left[\left(M_{g}^{2}+m_{g}^{2}\right)\left(A\left(e_{0}\right) B\left(e_{2}\right)+B\left(e_{0}\right) A\left(e_{2}\right)\right)\right.} \\
& \left.+\left(M_{g}^{2}-m_{g}^{2}\right)\left|A\left(e_{0}\right) B\left(e_{2}\right)-B\left(e_{0}\right) A\left(e_{2}\right)\right|-4 A\left(e_{1}\right) B\left(e_{1}\right) m_{g} M_{g}\right]^{\frac{1}{2}} .
\end{aligned}
$$

Proof Using the Cauchy-Schwarz inequality in (28), we have

$$
\begin{aligned}
\left|\mathcal{P}_{A, B}(f, g)\right| & \leq \frac{1}{2} \sqrt{A_{x} B_{y}\left(\left(x M_{f}-y m_{f}\right)^{2}\right)} \sqrt{A_{x} B_{y}\left(\left(x M_{g}-y m_{g}\right)^{2}\right)}, \\
\left|\mathcal{P}_{A, B}(f, g)\right| & \leq \frac{1}{2} \sqrt{A_{x} B_{y}\left(\left(x m_{f}-y M_{f}\right)^{2}\right)} \sqrt{A_{x} B_{y}\left(\left(x m_{g}-y M_{g}\right)^{2}\right)} .
\end{aligned}
$$


From the above we obtain

$$
\begin{aligned}
\left|\mathcal{P}_{A, B}(f, g)\right| \leq & \frac{1}{2} \sqrt{\max \left\{A_{x} B_{y}\left(\left(x M_{f}-y m_{f}\right)^{2}\right), A_{x} B_{y}\left(\left(x m_{f}-y M_{f}\right)^{2}\right)\right\}} \\
& \times \sqrt{\max \left\{A_{x} B_{y}\left(\left(x M_{g}-y m_{g}\right)^{2}\right), A_{x} B_{y}\left(\left(x m_{g}-y M_{g}\right)^{2}\right)\right\}} .
\end{aligned}
$$

Computing, we obtain

$$
\begin{aligned}
\max & \left\{A_{x} B_{y}\left(\left(x M_{f}-y m_{f}\right)^{2}\right), A_{x} B_{y}\left(\left(x m_{f}-y M_{f}\right)^{2}\right)\right\} \\
= & \frac{1}{2}\left[\left(M_{f}^{2}+m_{f}^{2}\right)\left(A\left(e_{0}\right) B\left(e_{2}\right)+B\left(e_{0}\right) A\left(e_{2}\right)\right)\right. \\
& \left.+\left(M_{f}^{2}-m_{f}^{2}\right)\left|A\left(e_{0}\right) B\left(e_{2}\right)-B\left(e_{0}\right) A\left(e_{2}\right)\right|-4 M_{f} m_{f} A\left(e_{1}\right) B\left(e_{1}\right)\right]
\end{aligned}
$$

and

$$
\begin{aligned}
\max & \left\{A_{x} B_{y}\left(\left(x M_{g}-y m_{g}\right)^{2}\right), A_{x} B_{y}\left(\left(x m_{g}-y M_{g}\right)^{2}\right)\right\} \\
= & \frac{1}{2}\left[\left(M_{g}^{2}+m_{g}^{2}\right)\left(A\left(e_{0}\right) B\left(e_{2}\right)+B\left(e_{0}\right) A\left(e_{2}\right)\right)\right. \\
& \left.+\left(M_{g}^{2}-m_{g}^{2}\right)\left|A\left(e_{0}\right) B\left(e_{2}\right)-B\left(e_{0}\right) A\left(e_{2}\right)\right|-4 M_{g} m_{g} A\left(e_{1}\right) B\left(e_{1}\right)\right] .
\end{aligned}
$$

So, we get

$$
\left|\mathcal{P}_{A, B}(f, g)\right| \leq \frac{1}{2} K_{f} \cdot \frac{1}{2} K_{g},
$$

where $K_{f}$ and $K_{g}$ are given in (30), respectively (31), which is the inequality from the conclusion.

A more general case is taken forward, which improves relationship (25).

Theorem 2.21 Let $f, g: D \rightarrow \mathbb{R}, D \subset[a, b], 0<a<b, f, g \in \mathcal{F}$. If there exist real numbers $m_{g}, M_{g}$ such that $m_{g} \leq g(x) \leq M_{g}, \forall x \in D$, then

$$
\left|\mathcal{P}_{A, B}(f, g)\right| \leq \frac{1}{2}\left|\mathcal{P}_{A, B}(f, f)\right|^{\frac{1}{2}} \cdot K_{g},
$$

where $K_{g}$ is given by (31).

Proof Using inequality (29), we have

$$
\left|\mathcal{P}_{A, B}(g, g)\right| \leq \frac{1}{4} K_{g}^{2}
$$

and replacing this in relation (16), we get inequality (24).

Remark 2.22 If we take $A(f)=B(f)=\int_{a}^{b} f(x) d x$, then we obtain

$$
\left|\mathcal{P}_{A, B}(f, g)\right| \leq \frac{1}{2 \sqrt{3}}\left|\mathcal{P}_{A, B}(f, f)\right|^{\frac{1}{2}}\left[2(b-a)\left(b^{3}-a^{3}\right)\left(M_{g}^{2}+m_{g}^{2}\right)-3 m_{g} M_{g}\left(b^{2}-a^{2}\right)^{2}\right]^{\frac{1}{2}},
$$

which is inequality (2.14) from [1, Th. 11]. 


\section{Applications}

In this section we investigate some new inequalities of Grüss type using Pompeiu's mean value theorem and the above results. Improvement of known inequalities is also given.

Theorem 3.1 Let $f, g:[a, b] \rightarrow \mathbb{R}, 0<a<b, f, g \in \mathcal{F}$. If $f \in C^{1}[a, b]$, then the following inequality

$$
\left|\mathcal{P}_{A, B}(f, g)\right| \leq \frac{1}{2} A_{x} B_{y}(|x-y| \cdot|y g(x)-x g(y)|)\left\|f-e_{1} f^{\prime}\right\|_{\infty}
$$

holds.

Proof From Lemma 1.4 we have that

$$
|y f(x)-x f(y)| \leq|x-y|\left\|f-e_{1} f^{\prime}\right\|_{\infty}
$$

and it follows that

$$
\begin{aligned}
\left|\mathcal{P}_{A, B}(f, g)\right| & =\frac{1}{2}\left|A_{x} B_{y}(y f(x)-x f(y))(y g(x)-x g(y))\right| \\
& \leq \frac{1}{2} A_{x} B_{y}(|x-y| \cdot|y g(x)-x g(y)|)|| f-e_{1} f^{\prime} \|_{\infty} .
\end{aligned}
$$

Remark 3.2 If we take $A(f)=B(f)=\int_{a}^{b} f(x) d x$, then from (29) we get the first result from [1, Th. 13].

Theorem 3.3 Let $f, g:[a, b] \rightarrow \mathbb{R}, 0<a<b, f, g \in \mathcal{F}$. If $f \in L^{1}[a, b]$, then the following inequality

$$
\left|\mathcal{P}_{A, B}(f, g)\right| \leq \frac{1}{2} A_{x} B_{y}\left(\frac{\max \{x, y\}}{\min \{x, y\}} \cdot|y g(x)-x g(y)|\right)\left\|f-e_{1} f^{\prime}\right\|_{1}
$$

holds.

Proof From Lemma 1.4 we have

$$
\begin{aligned}
\left|\mathcal{P}_{A, B}(f, g)\right| & =\frac{1}{2}\left|A_{x} B_{y}(y f(x)-x f(y))(y g(x)-x g(y))\right| \\
& \leq \frac{1}{2}\left\|f-e_{1} f^{\prime}\right\|_{1} A_{x} B_{y}\left(\frac{\max \{x, y\}}{\min \{x, y\}} \cdot|y g(x)-x g(y)|\right) .
\end{aligned}
$$

Remark 3.4 If we take $A(f)=B(f)=\int_{a}^{b} f(x) d x$, then from (36) we get the last result from [1, Th. 13].

Theorem 3.5 Let $f, g: D \rightarrow \mathbb{R}, D \subset[a, b], 0<a<b, f, g \in \mathcal{F}, f \in C^{1}[a, b]$. If there exist real numbers $m_{g}, M_{g}$ such that $m_{g} \leq g(x) \leq M_{g}, \forall x \in[a, b]$, then

$$
\begin{aligned}
\left|\mathcal{P}_{A, B}(f, g)\right| \leq & \frac{1}{2 \sqrt{2}}\left[B\left(e_{0}\right) A\left(e_{2}\right)+A\left(e_{0}\right) B\left(e_{2}\right)-2 A\left(e_{1}\right) B\left(e_{1}\right)\right]^{\frac{1}{2}} \\
& \times K_{g} \cdot\left\|f-e_{1} f^{\prime}\right\|_{\infty},
\end{aligned}
$$

where $K_{g}$ is given by (31). 
Proof From inequality (8) we get

$$
\left|\mathcal{P}_{A, B}(f, f)\right| \leq \frac{1}{2}\left[B\left(e_{0}\right) A\left(e_{2}\right)+A\left(e_{0}\right) B\left(e_{2}\right)-2 A\left(e_{1}\right) B\left(e_{1}\right)\right]\left\|f-e_{1} f\right\|_{\infty}^{2}
$$

On the other hand, from inequality (29) we have

$$
\begin{aligned}
\left|\mathcal{P}_{A, B}(g, g)\right| & \leq \frac{1}{4}\left[M_{g}^{2} A\left(e_{0}\right) B\left(e_{2}\right)+m_{g}^{2} A\left(e_{2}\right) B\left(e_{0}\right)-2 M_{g} m_{g} A\left(e_{1}\right) B\left(e_{1}\right)\right] \\
& =\frac{1}{4} K_{g} .
\end{aligned}
$$

Using the last two inequalities in (16), we get the conclusion.

Remark 3.6 If we take $A(f)=B(f)=\int_{a}^{b} f(x) d x$, then from (37) we get the result from [1, Th. 14].

Theorem 3.7 Let $f:[a, b] \rightarrow \mathbb{R}, 0<a<b, f, g \in \mathcal{F}, f \in C^{1}[a, b], g \in L^{1}[a, b]$. Then we have the following inequality:

$$
\begin{aligned}
\left|\mathcal{P}_{A, B}(f, g)\right| \leq & \frac{1}{4}\left\|f-e_{1} f^{\prime}\right\|_{\infty}\left\|g-e_{1} g^{\prime}\right\|_{1} \\
& \times\left[A\left(e_{0}\right) B\left(e_{2}\right)+A\left(e_{2}\right) B\left(e_{0}\right)-2 A\left(e_{1}\right) B\left(e_{1}\right)\right. \\
& \left.+\left|A\left(e_{2}\right) B\left(e_{0}\right)-A\left(e_{0}\right) B\left(e_{2}\right)\right|\right] .
\end{aligned}
$$

Proof From Lemma 1.4 we get

$$
\begin{aligned}
\left|\mathcal{P}_{A, B}(f, g)\right| & =\frac{1}{2}\left|A_{x} B_{y}(y f(x)-x f(y))(y g(x)-x g(y))\right| \\
& \leq \frac{1}{2}\left\|f-e_{1} f^{\prime}\right\|_{\infty}\left\|g-e_{1} g^{\prime}\right\|_{1} \cdot A_{x} B_{y}\left(\frac{\max \{x, y\}}{\min \{x, y\}} \cdot|x-y|\right) .
\end{aligned}
$$

If $x<y$, then

$$
\frac{\max \{x, y\}}{\min \{x, y\}} \cdot|x-y|=\frac{y}{x}(y-x)<y^{2}-x y .
$$

If $x \geq y$, then

$$
\frac{\max \{x, y\}}{\min \{x, y\}} \cdot|x-y|=\frac{x}{y}(x-y) \leq x^{2}-x y .
$$

Therefore, we have

$$
\begin{aligned}
& A_{x} B_{y}\left(\frac{\max \{x, y\}}{\min \{x, y\}} \cdot|x-y|\right) \\
& \leq \begin{cases}A_{x} B_{y}\left(y^{2}-x y\right), & \text { if } x<y, \\
A_{x} B_{y}\left(x^{2}-x y\right), & \text { if } x \geq y,\end{cases} \\
& \leq \max \left\{A_{x} B_{y}\left(y^{2}-x y\right), A_{x} B_{y}\left(x^{2}-x y\right)\right\}
\end{aligned}
$$




$$
\begin{aligned}
& =\max \left\{A\left(e_{0}\right) B\left(e_{2}\right)-A\left(e_{1}\right) B\left(e_{1}\right), A\left(e_{2}\right) B\left(e_{0}\right)-A\left(e_{1}\right) B\left(e_{1}\right)\right\} \\
& =\frac{1}{2}\left[A\left(e_{0}\right) B\left(e_{2}\right)+A\left(e_{2}\right) B\left(e_{0}\right)-2 A\left(e_{1}\right) B\left(e_{1}\right)+\left|A\left(e_{2}\right) B\left(e_{0}\right)-A\left(e_{0}\right) B\left(e_{2}\right)\right|\right] .
\end{aligned}
$$

Using the last inequality in (39), we obtain (38).

Remark 3.8 If we take $A(f)=B(f)=\int_{a}^{b} f(x) d x$, then inequality (39) becomes the following inequality:

$$
\left|\mathcal{P}_{A, B}(f, g)\right| \leq \frac{1}{24}(b-a)^{4}\left\|f-e_{1} f^{\prime}\right\|_{\infty}\left\|g-e_{1} g^{\prime}\right\|_{1} .
$$

Theorem 3.9 Let $f:[a, b] \rightarrow \mathbb{R}, 0<a<b, f, g \in \mathcal{F}, f \in C^{1}[a, b], g \in L^{1}[a, b]$. Then we have the following inequality:

$$
\left|\mathcal{P}_{A, B}(f, g)\right| \leq \frac{1}{2} A_{x} B_{y}\left(|x-y| \frac{\max \{x, y\}}{\min \{x, y\}}\right)\left\|f-e_{1} f^{\prime}\right\|_{\infty}\left\|g-e_{1} g^{\prime}\right\|_{1} .
$$

Proof From Lemma 1.4 we get

$$
\begin{aligned}
\left|\mathcal{P}_{A, B}(f, g)\right| & =\frac{1}{2}\left|A_{x} B_{y}(y f(x)-x f(y))(y g(x)-x g(y))\right| \\
& \leq \frac{1}{2} A_{x} B_{y}\left(|x-y| \frac{\max \{x, y\}}{\min \{x, y\}}\right)\left\|f-e_{1} f^{\prime}\right\|_{\infty}\left\|g-e_{1} g^{\prime}\right\|_{1} .
\end{aligned}
$$

Remark 3.10 If we take $A(f)=B(f)=\int_{a}^{b} f(x) d x$, then inequality (41) becomes inequality (3.5) from [1, Th. 15].

Theorem 3.11 Let $f:[a, b] \rightarrow \mathbb{R}, 0<a<b, f, g \in \mathcal{F}, f \in L^{1}[a, b]$. If there exist real numbers $m_{g}, M_{g}$ such that $m_{g} \leq g(x) \leq M_{g}, \forall x \in[a, b]$, then the following inequality holds:

$$
\left|\mathcal{P}_{A, B}(f, g)\right|=\frac{1}{2 \sqrt{2}}\left|A_{x} B_{y}\left(\left(\frac{\max \{x, y\}}{\min \{x, y\}}\right)^{2}\right)\right|^{\frac{1}{2}} \cdot K_{g} \cdot\left\|f-e_{1} f^{\prime}\right\|_{1}
$$

where $K_{g}$ is given by (31).

Proof From Lemma 1.4 we get

$$
\left|\mathcal{P}_{A, B}(f, f)\right| \leq \frac{1}{2}\left|A_{x} B_{y}\left(\frac{\max ^{2}\{x, y\}}{\min ^{2}\{x, y\}}\right)\right|\left\|f-e_{1} f^{\prime}\right\|_{1}^{2} .
$$

Using inequality (29), we have

$$
\left|\mathcal{P}_{A, B}(g, g)\right| \leq \frac{1}{4} K_{g}^{2}
$$

where $K_{g}$ is given by (31).

Substituting in (16) we get the desired result.

Remark 3.12 If we take $A(f)=B(f)=\int_{a}^{b} f(x) d x$, then inequality (42) becomes inequality (3.7) from [1, Th. 16]. 
Definition 3.13 Let $a, b \in \mathbb{R}$, with $a<b$ and $f, g, h \in \mathcal{F}, h:[a, b] \rightarrow \mathbb{R}_{+}$. The functional noted by $\mathcal{P}_{A, B}(f, g ; h)$, defined by

$$
\begin{aligned}
\mathcal{P}_{A, B}(f, g ; h)= & \frac{1}{2} A_{x} B_{y}\left[h^{2}(x) f(y) g(y)+h^{2}(y) f(x) g(x)\right. \\
& -(h(x) h(y) g(x) f(y)+h(x) h(y) f(x) g(y))],
\end{aligned}
$$

is called Pompeiu-Chebyshev with respect to the function $h$ functional.

Note that the functional can also be written in the following form:

$$
\mathcal{P}_{A, B}(f, g ; h)=\frac{1}{2} A_{x} B_{y}(h(x) f(y)-h(y) f(x))(h(x) g(y)-h(y) g(x)) .
$$

Definition 3.14 (See [1]) Let $f, g:[a, b] \rightarrow \mathbb{R}, f, g \in \mathcal{F}$. The functions $f$ and $g$ are called synchronous with respect to a function $h$ (h-synchronous, similarly ordered), $h:[a, b] \rightarrow$ $\mathbb{R}_{+}$, if for all $x, y \in[a, b]$, we have

$$
(h(x) f(y)-h(y) f(x))(h(x) g(y)-h(y) g(x)) \geq 0,
$$

and $f, g$ are called asynchronous with respect to a function $h$ ( $h$-asynchronous, oppositely ordered) if for all $x, y \in[a, b]$ we have

$$
(h(x) f(y)-h(y) f(x))(h(x) g(y)-h(y) g(x)) \leq 0 .
$$

The next result generalizes the inequalities from Theorem 2.7.

Theorem 3.15 Let $f, g:[a, b] \rightarrow \mathbb{R}, f, g \in \mathcal{F}$, and $h:[a, b] \rightarrow \mathbb{R}_{+}$such that $h(x) \neq 0, \forall x \in$ $[a, b]$.

(i) If $\frac{f}{h}$ and $\frac{g}{h}$ are h-synchronous functions, then

$$
\mathcal{P}_{A, B}(f, g ; h) \geq 0
$$

(ii) If $\frac{f}{h}$ and $\frac{g}{h}$ are h-asynchronous functions, then

$$
\mathcal{P}_{A, B}(f, g ; h) \leq 0
$$

Proof Since $\frac{f}{h}$ and $\frac{g}{h}$ are $h$-synchronous (h-asynchronous) functions, we have

$$
(h(x) f(y)-h(y) f(x))(h(x) g(y)-h(y) g(x)) \geq(\leq) 0, \quad \forall x, y \in[a, b] .
$$

From this and (43) we have

$$
\mathcal{P}_{A, B}(f, g ; h)=\frac{1}{2} A_{x} B_{y}(h(x) f(y)-h(y) f(x))(h(x) g(y)-h(y) g(x)) \geq(\leq) 0,
$$

from where we get the conclusion. 
Remark 3.16 In (46), respectively (47), if we take $h(x)=x, x \in[a, b]$, then we obtain the inequalities from Theorem 2.7.

Remark 3.17 If we take $A(f)=B(f)=\int_{a}^{b} f(x) d x$, then inequality (46) becomes inequality (4.5) from [1, Th. 19].

The next theorem is a generalization of Theorem 2.9 and contains the pre-Grüss inequality.

Theorem 3.18 Let $f, g:[a, b] \rightarrow \mathbb{R}, a, b \in \mathbb{R}, a<b$, and $f, g \in \mathcal{F}$. If $h \in \mathcal{F}$ is a positive function, then

$$
\left|\mathcal{P}_{A, B}(f, g ; h)\right| \leq\left|\mathcal{P}_{A, B}(f, f ; h)\right|^{\frac{1}{2}}\left|\mathcal{P}_{A, B}(g, g ; h)\right|^{\frac{1}{2}}
$$

Proof Using the CBS inequality in equality (43), we obtain

$$
\begin{aligned}
\left|\mathcal{P}_{A, B}(f, g ; h)\right| \leq & {\left[\frac{1}{2} A_{x} B_{y}(h(x) f(y)-h(y) f(x))^{2}\right]^{\frac{1}{2}} } \\
& \times\left[\frac{1}{2} A_{x} B_{y}(h(x) g(y)-h(y) g(x))^{2}\right]^{\frac{1}{2}} .
\end{aligned}
$$

But we have

$$
\frac{1}{2} A_{x} B_{y}(h(x) f(y)-h(y) f(x))^{2}=\mathcal{P}_{A, B}(f, f ; h)
$$

and

$$
\frac{1}{2} A_{x} B_{y}(h(x) g(y)-h(y) g(x))^{2}=\mathcal{P}_{A, B}(g, g ; h) .
$$

From the above we get the conclusion.

Remark 3.19 In (46), if we take $h(x)=x, x \in[a, b]$, then we obtain inequality (16) from Theorem 2.9.

Remark 3.20 If we take $A(f)=B(f)=\int_{a}^{b} f(x) d x$, then inequality (46), respectively (47), becomes inequalities (4.8) from [1, Corollary 8$]$.

It is easy to see that the Pompeiu-Chebyshev functional with respect to the function $h$, $\mathcal{P}_{A, B}(f, f ; h)$ represents the reverse of CBS-inequality. We have

$$
\mathcal{P}_{A, B}(f, f ; h)=\frac{1}{2} A_{x} B_{y}\left((h(x) f(y)-h(y) f(x))^{2}\right) \geq 0 .
$$

We recall that a function $f:[a, b] \rightarrow \mathbb{R}$ is called of $p-H$-Hölder type, with $H>0, p \in(0,1]$, if for any $x, y \in[a, b]$ we have

$$
|f(x)-f(y)| \leq H|x-y|^{p} .
$$

In [2], Barnett and Dragomir proved the following theorem. 
Theorem 3.21 If $f, g$ are measurable on $[a, b]$ and $\frac{f}{g}$ is $p-H$-Hölder type, with $H>0$, $p \in(0,1])$, then

$$
\begin{aligned}
0 & \leq \int_{a}^{b} f^{2}(x) d x \int_{a}^{b} g^{2}(x) d x-\left(\int_{a}^{b} f(x) g(x) d x\right)^{2} \\
& \leq H^{2}\left\{\begin{array}{l}
\frac{(b-a)^{2 p+2}}{(2 p+1)(2 p+2)}\|h\|_{\infty}^{4}, \quad \text { if } h \in L_{\infty}[a, b], \\
\frac{2^{-\frac{1}{\beta}}(b-a)^{2 p+\frac{2}{\alpha}}}{(2 \alpha p+1)^{\frac{1}{\alpha}}(2 \alpha p+2)^{\frac{1}{\alpha}}}\|h\|_{2 \beta}^{4}, \quad \text { if } h \in L_{2 \beta}[a, b], \\
\frac{1}{2}(b-a)^{2 p}\|h\|_{2}^{4}, \quad \text { if } h \in L_{2}[a, b],
\end{array}\right.
\end{aligned}
$$

for $\alpha, \beta>1, \frac{1}{\alpha}+\frac{1}{\beta}=1$ and $\|h\|_{p}=\left(\int_{a}^{b}|f(x)|^{p} d x\right)^{\frac{1}{p}}$.

Starting from this we can state the following results.

Theorem 3.22 Let $a, b \in \mathbb{R}, a<b$, and $f, g, h \in \mathcal{F}$, where $f, g, h:[a, b] \rightarrow \mathbb{R}$. If $\frac{f}{h}$ and $\frac{g}{h}$ are of $p-H$-Hölder type, with $H_{1}, H_{2}>0, p, q \in(0,1]$, then

$$
\left|\mathcal{P}_{A, B}(f, g ; h)\right| \leq \frac{1}{2} H_{1} H_{2} \cdot A_{x} B_{y}\left(|x-y|^{p+q} h^{2}(x) h^{2}(y)\right) .
$$

Proof From $\frac{f}{h}$ and $\frac{g}{h}$ are of $p-H$-Hölder type with $H_{1}, H_{2}>0, p, q \in(0,1]$ we have

$$
\left|\frac{f(x)}{h(x)}-\frac{f(y)}{h(y)}\right| \leq H_{1}|x-y|^{p}, \quad \forall x, y \in[a, b]
$$

and

$$
\left|\frac{g(x)}{h(x)}-\frac{g(y)}{h(y)}\right| \leq H_{2}|x-y|^{q}, \quad \forall x, y \in[a, b] .
$$

By multiplying the last two inequalities, we obtain

$$
\frac{|h(x) f(y)-h(y) f(x)| \cdot|h(x) g(y)-h(y) g(x)|}{|h(x) h(y)|^{2}} \leq H_{1} H_{2}|x-y|^{p+q}
$$

Using (43) in the last inequality $\mathcal{P}_{A, B}(f, g ; h)$, we obtain the conclusion.

Remark 3.23 If we take $A(f)=B(f)=\int_{a}^{b} f(x) d x$, then inequality (41) becomes inequality (4.13) from [1, Theorem 20], which represents the following inequalities:

$$
\begin{aligned}
\left|\mathcal{P}_{A, B}(f, g ; h)\right| \leq & H_{1} H_{2} \\
& \times\left\{\begin{array}{l}
\frac{(b-a)^{p+q+2}}{\sqrt{(2 p+1)(2 p+2)(2 q+1)(2 q+2)}}\|h\|_{\infty}^{4}, \quad \text { if } h \in L_{\infty}[a, b], \\
\frac{2^{-\frac{1}{\beta}}(b-a)^{p+q+\frac{2}{\alpha}}}{[(2 \alpha p+1)(2 \alpha p+2)(2 \alpha q+1)(2 \alpha q+2)]^{\frac{1}{\alpha}}}\|h\|_{2 \beta}^{4}, \quad \text { if } h \in L_{2 \beta}[a, b], \\
\frac{1}{2}(b-a)^{p+q}\|h\|_{2}^{4}, \quad \text { if } h \in L_{2}[a, b] .
\end{array}\right.
\end{aligned}
$$


Theorem 3.24 Let $a, b \in \mathbb{R}, a<b$, and $f, g, h \in \mathcal{F}$, where $f, g, h:[a, b] \rightarrow \mathbb{R}$. If $\frac{h}{f}$ and $\frac{h}{g}$ are of $p$-H-Hölder type, with $H_{1}, H_{2}>0, p, q \in(0,1]$, then

$$
\left|\mathcal{P}_{A, B}(f, g ; h)\right| \leq \frac{1}{2} H_{1} H_{2} \cdot A_{x} B_{y}\left(|x-y|^{p+q} f(x) f(y) g(x) g(y)\right) .
$$

Proof From $\frac{h}{f}$ and $\frac{h}{g}$ are of $p-H$-Hölder type with $H_{1}, H_{2}>0, p, q \in(0,1]$ we have

$$
\left|\frac{h(x)}{f(x)}-\frac{h(y)}{f(y)}\right| \leq H_{1}|x-y|^{p}, \quad \forall x, y \in[a, b]
$$

and

$$
\left|\frac{h(x)}{g(x)}-\frac{h(y)}{g(y)}\right| \leq H_{2}|x-y|^{q}, \quad \forall x, y \in[a, b] .
$$

By multiplying the last two inequalities, we obtain

$$
\frac{|h(x) f(y)-h(y) f(x)| \cdot|h(x) g(y)-h(y) g(x)|}{|f(x) f(y) g(x) g(y)|} \leq H_{1} H_{2}|x-y|^{p+q} .
$$

Applying in the last inequality $\mathcal{P}_{A, B}(f, g ; h)$, we obtain the conclusion.

Remark 3.25 If we take $A(f)=B(f)=\int_{a}^{b} f(x) d x$, then inequality (43) becomes (4.16) from [1, Theorem 21], which represents the following inequalities:

$$
\begin{aligned}
\left|\mathcal{P}_{A, B}(f, g ; h)\right| \leq & H_{1} H_{2} \\
& \times\left\{\begin{array}{l}
\frac{(b-a)^{p+q+2}}{\sqrt{(2 p+1)(2 p+2)(2 q+1)(2 q+2)}}\|f\|_{\infty}^{2}\|g\|_{\infty}^{2}, \quad f, g \in L_{\infty}[a, b], \\
\frac{2^{-\frac{1}{\beta}(b-a)^{p+q+\frac{2}{\alpha}}}}{[(2 \alpha p+1)(2 \alpha p+2)(2 \alpha q+1)(2 \alpha q+2)]^{\frac{1}{\alpha}}}\|f\|_{2 \beta}^{2}\|g\|_{2 \beta}^{2}, \quad f, g \in L_{2 \beta}[a, b], \\
\frac{1}{2}(b-a)^{p+q}\|f\|_{2}^{2}\|g\|_{2}^{2}, \quad f, g \in L_{2}[a, b] .
\end{array}\right.
\end{aligned}
$$

\section{Examples}

In this section we give some examples by choosing the functionals $A(f)$ and $B(f)$ in different forms and, in this way, we obtain some inequalities.

Example 4.1 Let

$$
A(f)=\frac{1}{b-a} \int_{a}^{b} f(x) d x \quad \text { and } \quad B(f)=\frac{f(a)+f(b)}{2}
$$

be two functionals for which we have

$$
A\left(e_{0}\right)=1, \quad A\left(e_{1}\right)=\frac{a+b}{2}, \quad A\left(e_{2}\right)=\frac{a^{2}+a b+b^{2}}{3}
$$

and

$$
B\left(e_{0}\right)=1, \quad B\left(e_{1}\right)=\frac{a+b}{2}, \quad B\left(e_{2}\right)=\frac{a^{2}+b^{2}}{2} .
$$


Using (7) for the functionals $A$ and $B$ chosen, we obtain

$$
\begin{aligned}
\mathcal{P}_{A, B}(f, g)= & \frac{1}{2}\left[\frac{a^{2}+b^{2}}{2(b-a)} \int_{a}^{b} f(x) g(x) d x+\frac{a^{2}+a b+b^{2}}{3} \cdot \frac{f(a) g(a)+f(b) g(b)}{2}\right. \\
& \left.-\frac{a g(a)+b g(b)}{2(b-a)} \int_{a}^{b} x f(x) d x-\frac{a f(a)+b f(b)}{2(b-a)} \int_{a}^{b} x g(x) d x\right] .
\end{aligned}
$$

For the functional defined by (56), we obtain the following inequalities:

(a) If $0<a<b, f, g:[a, b] \rightarrow \mathbb{R}, f, g \in \mathcal{F}, f, g \in C^{1}[a, b]$, then

$$
\left|\mathcal{P}_{A, B}(f, g)\right| \leq \frac{(b-a)^{2}}{6}\left\|f-e_{1} f^{\prime}\right\|_{\infty}\left\|g-e_{1} g^{\prime}\right\|_{\infty}
$$

(b) If $0<a<b, f, g:[a, b] \rightarrow \mathbb{R}, f, g \in \mathcal{F}$, and there exist real numbers $m_{f}, M_{f}, m_{g}, M_{g}$ such that $m_{f} \leq f(x) \leq M_{f}$ and $m_{g} \leq g(x) \leq M_{g}, \forall x \in D$, then

$$
\left|\mathcal{P}_{A, B}(f, g)\right| \leq \frac{(b-a)^{2}}{6}\left(b M_{f}-a m_{f}\right)\left(b M_{g}-a m_{g}\right)
$$

Example 4.2 Let

$$
R_{\alpha}(f)=\frac{1}{\Gamma(\alpha)} \int_{a}^{b}(b-x)^{\alpha-1} f(x) d x, \quad \alpha \in \mathbb{N}, \alpha \geq 1
$$

be a Riemann-Liouville type functional for which we have

$$
\begin{aligned}
& R_{\alpha}\left(e_{0}\right)=\frac{(b-a)^{\alpha}}{\alpha !}, \\
& R_{\alpha}\left(e_{1}\right)=\frac{(a \alpha+b)(b-a)^{\alpha}}{(\alpha+1) !} \\
& R_{\alpha}\left(e_{2}\right)=\frac{a^{2}(b-a)^{\alpha}}{\alpha !}+\frac{2 a(b-a)^{\alpha+1}}{(\alpha+1) !}+\frac{2(b-a)^{\alpha+2}}{(\alpha+2) !} .
\end{aligned}
$$

For $\alpha=1$ we denote

$$
R_{1}(f)=A(f)=\int_{a}^{b} f(x) d x
$$

and for $\alpha=2$ we denote

$$
R_{2}(f)=B(f)=\int_{a}^{b}(b-x) f(x) d x
$$

We have

$$
A\left(e_{0}\right)=b-a, \quad A\left(e_{1}\right)=\frac{b^{2}-a^{2}}{2}, \quad A\left(e_{2}\right)=\frac{b^{3}-a^{3}}{3}
$$

and

$$
B\left(e_{0}\right)=\frac{(b-a)^{2}}{2}
$$




$$
\begin{aligned}
& B\left(e_{1}\right)=\frac{(2 a+b)(b-a)^{2}}{6}, \\
& B\left(e_{2}\right)=\frac{\left(3 a^{2}+b^{2}+2 a b\right)(b-a)^{2}}{12} .
\end{aligned}
$$

Substituting (60) and (61) in (7), we obtain

$$
\begin{aligned}
\mathcal{P}_{A, B}(f, g)= & \frac{1}{2}\left[\frac{\left(3 a^{2}+b^{2}+2 a b\right)(b-a)^{2}}{12} \int_{a}^{b} f(x) g(x) d x\right. \\
& +\frac{b^{3}-a^{3}}{3} \int_{a}^{b}(b-x) f(x) g(x) d x-\int_{a}^{b} x f(x) d x \int_{a}^{b}(b-x) x g(x) d x \\
& \left.-\int_{a}^{b} x g(x) d x \int_{a}^{b}(b-x) x f(x) d x\right] .
\end{aligned}
$$

For the functional defined by (62), we obtain the following inequalities:

(a) If $0<a<b, f, g:[a, b] \rightarrow \mathbb{R}, f, g \in \mathcal{F}, f, g \in C^{1}[a, b]$, then

$$
\left|\mathcal{P}_{A, B}(f, g)\right| \leq \frac{(b-a)^{5}}{24}\left\|f-e_{1} f^{\prime}\right\|_{\infty}\left\|g-e_{1} g^{\prime}\right\|_{\infty} .
$$

(b) If $0<a<b, f, g:[a, b] \rightarrow \mathbb{R}, f, g \in \mathcal{F}$, and there exist real numbers $m_{f}, M_{f}, m_{g}, M_{g}$ such that $m_{f} \leq f(x) \leq M_{f}$ and $m_{g} \leq g(x) \leq M_{g}, \forall x \in[a, b]$, then

$$
\left|\mathcal{P}_{A, B}(f, g)\right| \leq \frac{(b-a)^{5}}{24}\left(b M_{f}-a m_{f}\right)\left(b M_{g}-a m_{g}\right)
$$

(c) If $0<a<b, f, g:[a, b] \rightarrow \mathbb{R}, f, g \in \mathcal{F}$, and there exist real numbers $m_{g}, M_{g}$ such that $m_{g} \leq g(x) \leq M_{g}, \forall x \in[a, b]$, then

$$
\left|\mathcal{P}_{A, B}(f, g)\right| \leq \frac{(b-a)^{\frac{5}{2}}}{2 \sqrt{6}}\left|\mathcal{P}_{A, B}(f, g)\right|^{\frac{1}{2}}\left(b M_{g}-a m_{g}\right)
$$

Example 4.3 Let

$$
R_{\alpha}(f)=\frac{1}{\Gamma(\alpha)} \int_{a}^{b}(b-x)^{\alpha-1} f(x) d x, \quad \alpha>0
$$

and

$$
R_{\beta}(f)=\frac{1}{\Gamma(\beta)} \int_{a}^{b}(b-x)^{\beta-1} f(x) d x, \quad \beta>0
$$

be two Riemann-Liouville type functionals for which we have

$$
\begin{aligned}
& R_{\varphi}\left(e_{0}\right)=\frac{(b-a)^{\varphi}}{\varphi !}, \quad R_{\varphi}\left(e_{1}\right)=\frac{(\varphi a+b)(b-a)^{\varphi}}{(\varphi+1) !} \\
& R_{\varphi}\left(e_{2}\right)=\frac{a^{2}(b-a)^{\varphi}}{\varphi !}+\frac{2 a(b-a)^{\varphi+1}}{(\varphi+1) !}+\frac{2(b-a)^{\varphi+2}}{(\varphi+2) !}
\end{aligned}
$$

where $\varphi \in\{\alpha, \beta\}$. 
For $R_{\alpha}(f)=A(f)$ and $R_{\beta}(f)=B(f)$, using relations (66) in (7), we have

$$
\begin{aligned}
\mathcal{P}_{A, B}(f, g) & =\frac{1}{2}\left[\left(\frac{a^{2}(b-a)^{\beta}}{\beta !}+\frac{2 a(b-a)^{\beta+1}}{(\beta+1) !}+\frac{2(b-a)^{\beta+2}}{(\beta+2) !}\right)\right. \\
& \times \frac{1}{\Gamma(\alpha)} \int_{a}^{b}(b-x)^{\alpha-1} f(x) g(x) d x \\
& +\left(\frac{a^{2}(b-a)^{\alpha}}{\alpha !}+\frac{2 a(b-a)^{\alpha+1}}{(\alpha+1) !}+\frac{2(b-a)^{\alpha+2}}{(\alpha+2) !}\right) \frac{1}{\Gamma(\beta)} \int_{a}^{b}(b-x)^{\beta-1} f(x) g(x) d x \\
& -\frac{1}{\Gamma(\alpha) \Gamma(\beta)} \int_{a}^{b}(b-x)^{\alpha-1} x f(x) d x \int_{a}^{b}(b-x)^{\beta-1} x g(x) d x \\
& \left.-\frac{1}{\Gamma(\alpha) \Gamma(\beta)} \int_{a}^{b}(b-x)^{\beta-1} x f(x) d x \int_{a}^{b}(b-x)^{\alpha-1} x g(x) d x\right] .
\end{aligned}
$$

For the functional defined by (67), we obtain the following inequalities:

(a) If $0<a<b, f, g:[a, b] \rightarrow \mathbb{R}, f, g \in \mathcal{F}, f, g \in C^{1}[a, b]$, then

$$
\left|\mathcal{P}_{A, B}(f, g)\right| \leq \frac{\left(\alpha^{2}+\beta^{2}+\alpha+\beta-\alpha \beta\right)(b-a)^{\alpha+\beta+2}}{(\alpha+2) !(\beta+2) !}\left\|f-e_{1} f^{\prime}\right\|_{\infty}\left\|g-e_{1} g^{\prime}\right\|_{\infty}
$$

(b) If $0<a<b, f, g:[a, b] \rightarrow \mathbb{R}, f, g \in \mathcal{F}$, and there exist real numbers $m_{f}, M_{f}, m_{g}, M_{g}$ such that $m_{f} \leq f(x) \leq M_{f}$ and $m_{g} \leq g(x) \leq M_{g}, \forall x \in[a, b]$, then

$$
\begin{aligned}
\left|\mathcal{P}_{A, B}(f, g)\right| \leq & \frac{\left(\alpha^{2}+\beta^{2}+\alpha+\beta-\alpha \beta\right)(b-a)^{\alpha+\beta+2}}{(\alpha+2) !(\beta+2) !} \\
& \times\left(b M_{f}-a m_{f}\right)\left(b M_{g}-a m_{g}\right) .
\end{aligned}
$$

(c) If $0<a<b, f, g:[a, b] \rightarrow \mathbb{R}, f, g \in \mathcal{F}$, and there exist real numbers $m_{g}, M_{g}$ such that $m_{g} \leq g(x) \leq M_{g}, \forall x \in[a, b]$, then

$$
\begin{aligned}
\left|\mathcal{P}_{A, B}(f, g)\right| \leq & {\left[\frac{\left(\alpha^{2}+\beta^{2}+\alpha+\beta-\alpha \beta\right)(b-a)^{\alpha+\beta+2}}{(\alpha+2) !(\beta+2) !}\right]^{\frac{1}{2}} } \\
& \times\left|\mathcal{P}_{A, B}(f, f)\right|^{\frac{1}{2}}\left(b M_{g}-a m_{g}\right) .
\end{aligned}
$$

\section{Acknowledgements}

The authors are grateful to the PhD coordinator, Prof. Ioan Gavrea, Department of Mathematics, Technical University of Cluj-Napoca, Romania for his careful reading of the manuscript and recommendations which improved the quality of the paper.

\section{Funding}

Not applicable.

Availability of data and materials

Not applicable.

Competing interests

The authors declare that they have no competing interests. 


\section{Publisher's Note}

Springer Nature remains neutral with regard to jurisdictional claims in published maps and institutional affiliations.

Received: 15 November 2019 Accepted: 12 February 2020 Published online: 24 February 2020

\section{References}

1. Alomari, M.W.: On Pompeiu-Chebyshev functional and its generalization. Results Math. 74, 1-36 (2019)

2. Barnett, N.S., Dragomir, S.S.: An additive reverse of the Cauchy-Bunyakovsky-Schwarz integral inequality. Appl. Math. Lett. 21, 388-393 (2008)

3. Chebysev, P.L.: Sur les expressions approximatives des integrals definis parles outres prises entre les meme limites. Proc. Math. Soc. Charkov 2, 93-98 (1882)

4. Dragomir, S.S.: Some Grüss-type results via Pompeiu-like inequalities. Arab. J. Math. 4, 159-170 (2015)

5. Grüss, G.: Uber das maximum des absoluten Betrages von $\frac{1}{b-a} \int_{a}^{b} f(x) g(x) d x-\frac{1}{(b-a)^{2}} \int_{a}^{b} f(x) d x \int_{a}^{b} g(x) d x$. Math. Z. 39, 215-226 (1935)

6. Lupaş, A.: The best constant in an integral inequality. Mathematica 15(38), 219-222 (1973)

7. Ostrowski, A.M.: On an integral inequality. Aequ. Math. 4, 358-373 (1970)

8. Pachpatte, B.G.: On Grüss like integral inequalities via Pompeiu's mean value theorem. J. Inequal. Pure Appl. Math. 6(3), $132(2005)$

9. Pompeiu, D.: Sur une proposition analogue au theoreme des accroissements finis. Mathematica 22, 143-146 (1946)

\section{Submit your manuscript to a SpringerOpen ${ }^{\circ}$ journal and benefit from:}

- Convenient online submission

- Rigorous peer review

- Open access: articles freely available online

- High visibility within the field

- Retaining the copyright to your article

Submit your next manuscript at $\gg$ springeropen.com 